\title{
The Use of Snake Venom Toxins as Tools to Study Platelet Receptors for Collagen and von Willebrand Factor
}

\author{
Robert K. Andrews ${ }^{\mathrm{e}}$ Aura S. Kamiguti ${ }^{\mathrm{b}}$ Oscar Berlanga ${ }^{\mathrm{a}} \quad$ Mireille Leduc $^{\mathrm{c}}$ \\ R. David G. Theakston ${ }^{d}$ Steve P. Watson ${ }^{a}$ \\ a Departments of Pharmacology, University of Oxford, Oxford, bDepartment of Haematology, \\ Royal University Hospital, University of Liverpool, Liverpool, UK; ' 'L'Unité des Venins, \\ Institut Pasteur, Paris, France; dAlistair Reid Venom Research Unit, Liverpool School of

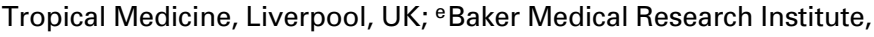 \\ Melbourne, Vic., Australia
}

\section{Key Words}

Collagen · Convulxin · von Willebrand factor · GPVI - GPIb-IX-V · Snake toxin • C-type lectin $\cdot$ Metalloproteinase $\cdot$ Botrocetin

\begin{abstract}
A large proportion of the biologically active proteins and peptides present within snake venoms interact with components of the haemostatic system to promote or inhibit the normal sequence of events that lead to clot formation. The venom proteins achieve their effects through interaction with various components of the coagulation cascade, endothelial matrix and platelets. Within the latter group, a number of venom proteins target the interaction of platelets with the major adhesive proteins, von Willebrand factor and
\end{abstract}

\begin{tabular}{ll}
\hline KARGER & ( ) 2002 S. Karger AG, Basel \\
0301-0147/01/0316-0155\$17.50/0 \\
$\begin{array}{l}\text { E-Mail karger@karger.ch } \\
\text { www.karger.com }\end{array}$ & $\begin{array}{l}\text { Accessible online at: } \\
\text { www.karger.com/journals/hae }\end{array}$
\end{tabular}

collagen. The venom proteins bind either the adhesive protein itself or their receptors on the platelet surface, notably GP-Ib-IX-V and GPVI. This review discusses the substantial contribution that venom proteins have made to our understanding of the role of these two adhesive proteins and their receptors (excluding GPIIb-IIla) in platelet regulation.

Copyright $\odot 2002$ S. Karger AG, Basel

\section{Introduction}

Snake venoms contain a wide variety of biologically active components that enable the snake to paralyse, kill and digest its prey. Generally, venomous snakes are divided into two major categories depending on whether their venom has predominantly neurotoxic or
Steve P. Watson

Department of Pharmacology, University of Oxford

Mansfield Road

Oxford OX1 3QT (UK)

E-Mail steve.watson@pharm.ox.ac.uk 
vascular effects. Among those with vascular effects, the result can be either prolonged bleeding or accelerated clotting, the latter resulting in fibrinogen consumption and often incoagulable blood. Although the former is more common, both effects frequently occur concurrently following a bite. The active components react with coagulation factors, platelets, endothelial cells and the extracellular matrix. The major classes of venom proteins which interact with platelets include metalloproteinases, phospholipases, disintegrins and C-type lectins.

\section{Venomous Snakes}

The prime aim of snake venom is the rapid immobilisation of the prey, whilst a secondary purpose is defence. Snake bite in humans is mainly a rural and occupational hazard and, as such, farmers and landworkers are at greatest risk. Although death frequently results from snake bite, more commonly (and fortunately) little or no envenoming occurs because only a small amount or no venom is injected. On the other hand, mortality can be high when adequate medical treatment is not given for severe systemic envenoming; it can be as high as $50 \%$ in seasnake envenoming and $10-15 \%$ in envenoming by the viper Echis ocellatus in West Africa.

Venomous snakes are divided into three major groups: elapids (F. Elapidae; e.g. cobras, mambas, kraits, coral snakes), seasnakes (F. Hydrophiidae) and vipers (F. Viperidae). The Viperidae is subdivided into the true vipers (SF. Viperinae; e.g. Russell's viper, carpet viper, puff adder) and the pit vipers (SF. Crotalinae; rattlesnakes, green tree vipers, Malayan pit viper, jararaca). There is also a small group of venomous colubrids (F. Colubridae; back-fanged snakes), which includes the Boomslang (Dispholidus typus) of Africa.

156

Haemostasis 2001;31:155-172

\section{Venoms}

Venoms from snakes contain a complex mixture of toxins, mainly proteins, which can be experimentally fractionated into enzymes and polypeptides with widely varying biological, pharmacological and autopharmacological properties; they also contain low molecular weight components which have not yet been thoroughly investigated. The toxic effects depend on both the type and amount of venom injected. Although viper venoms mainly cause haemorrhage and coagulation disorders, and elapid venoms cause neurotoxicity, there are exceptions to this rule and one should be wary of an excessively dogmatic classification.

Procoagulant enzymes, present mainly in venoms of vipers but also in that of a few elapids, stimulate blood coagulation by activating different steps of the coagulation cascade. The result in envenomed humans is fibrinogen consumption and therefore incoagulable blood (consumption coagulopathy), whereas in a small prey animal, massive intravascular coagulation causes rapid immobilisation and death. Zinc metalloproteinase haemorrhagins, also present mainly in viper venoms, damage the endothelium and subendothelium of the blood vessel walls, causing both local and systemic haemorrhage. Many venoms possess cytolytic or necrotising toxins; these are digestive hydrolases (proteolytic enzymes and phospholipases $\mathrm{A}_{2}$ ), polypeptide toxins and other factors which increase permeability, resulting in local swelling, as well as causing tissue breakdown usually in the region of the bite site. Haemolytic and myolytic phospholipases $\mathrm{A}_{2}$ are often also present which result in damage to cell membranes, endothelium, skeletal muscle, nerves and erythrocytes. Postsynaptic neurotoxins, present in the venom of many elapids, such as cobras, compete with acetylcholine for receptors in the neuromuscular junction and lead to cu- 


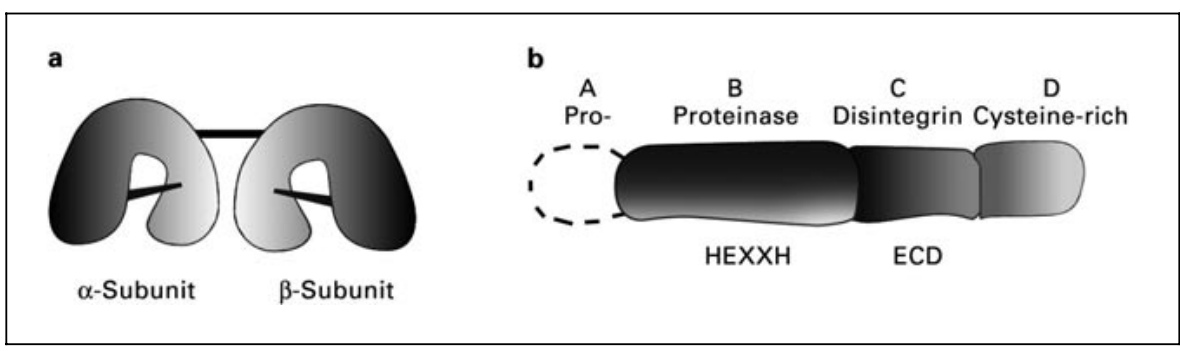

Fig. 1. a Structural representation of the $25-\mathrm{kD}$ form of the $\alpha \beta$ heterodimeric proteins of the snake venom C-type lectin-like family. Only major inter- and intra-subunit disulphide bonds common to several forms are shown (solid lines). b Metalloproteinase-disintegrin family proteins. The domain structure of a form lacking the pro-peptide domain (dashed line) is shown. HEXXH, His-Glu-X-X-His and ECD, Glu-Cys-Asp are sequences common to many forms.

rare-like paralysis. Presynaptic neurotoxins, present mainly in elapids but also in a few viperids, damage nerve endings, releasing acetylcholine transmitter or interfering with its release.

Over the years, snake venoms, especially those from certain viperine snakes, have been shown to contain a large number of toxins that exert powerful inhibitory and stimulatory effects on platelets. Several of these have made significant contributions to the understanding of the events underlying platelet activation. The purpose of this article is to consider the use of snake venom toxins as tools to study receptors for two of the major platelet adhesive proteins, collagen and von Willebrand factor, where extensive advances have been made within the last few years. For a more widespread discussion of the action of snake venoms on the haemostatic system, the reader is referred to a recent, extensive review [1].

Structural Classes of Snake Venom Toxins The snake venom proteins that interfere with the action of adhesion proteins on platelets fall into three distinct structural classes, namely snake C-type lectins, disintegrins and metalloproteinases. The metalloproteinases and C-type lectins may have evolved from a common precursor [1]. The disintegrins target the major platelet aggregatory receptor, GPIIb-IIIa $\left(\alpha_{\text {IIb }} \beta_{3}\right)$. The disintegrin domain is also found in a number of metalloproteinases where it has a distinct recognition site of ECD rather than RGD and is unable to bind GPIIb-IIIa. The structural organization of snake C-type lectins and a metalloproteinasedisintegrin is shown in figure 1.

C-Type Lectins. C-type lectins were first described as carbohydrate-binding (hence lectin) and calcium-dependent (hence C-type). They contain a carbohydrate recognition domain (CRD) of 130 amino acids, which reveals a series of invariant amino acid residues in a characteristic pattern $[2,3]$. In this pattern, four cysteines form intracatenary disulphide bridges.

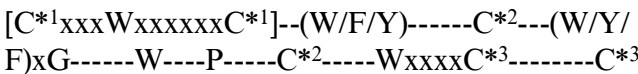
(the cysteines are disulphide linked to the cysteine with the same number). 
Recent crystallographic analyses of C-type lectins show: (1) amino acid residues involved in the carbohydrate binding are located in the C-terminal hydrophilic region which forms a peripheral loop; (2) critical amino acid residues define carbohydrate-binding specificity of C-type lectins: EPN for the mannose-specific motif and QPD for the galactose-specific motif; $\mathrm{Ca}^{2+}$ is bound to the motif Q-D in galactose-specific lectins $[4,5]$. The snake venom class of C-type lectins, however, do not bind sugars nor require calcium, but have other strong similarities with typical lectin domains.

During the last decade, several C-type snake proteins have been cloned and sequenced. Snake venom C-type lectins are found in true vipers and pit vipers but not in elapids and seasnakes [6]. They are made up of homodimers and heterodimers, often present as multiples. Amino acid sequences of each subunit contain between 125 and 135 residues, which corresponds to the CRD length proposed by Drickamer [2]. The amino acid identity between the snake C-type lectins is $50 \%$ or higher [7]. In all of these venoms, the sequences were found to contain the invariant amino acids of CRD as proposed by Spiess [3], with cysteine residues forming intra-disulphide bridges as chemically confirmed in botrocetin [8], isolated from the venom of Bothrops jararaca. Only the sequences of the homodimer of the rattlesnake lectin from Crotalus atrox and LmsL from Lachesis muta stenophyrs contain the amino acids in their CRD for binding galactose and $\mathrm{Ca}^{2+}$. The subunits of the heterodimeric proteins do not contain these amino acids and do not bind mannose or galactose [9]. However, in some cases, these heterodimeric proteins are able to bind $\mathrm{Ca}^{2+}$, and this has been shown to be necessary for their biological activity.

On the basis of the crystal structure of mannose-binding protein, the crystal struc- ture of several snake venom C-type lectins have been reported including factor IX/factor X-binding protein [10], factor IX-binding protein [11], botrocetin [12] and flavocetin A [12]. These crystal structures show that dimerisation of their CRDs is an example of loop-swapping. It has been proposed that this is responsible for their loss of carbohydrate binding activity. The crystal structures also confirmed the absence of a canonical $\mathrm{Ca}^{2+}$ binding site, but revealed the presence of a new one localized in the C-type lectin fold. Such dimerisation of the CRD in the snake venom toxin was proposed as a strategy for survival against animal predators and exemplifies an evolutionary gain of function for the C-type lectin domain.

Disintegrins. These are cysteine-rich venom components $(5-10 \mathrm{kD})$ with potent inhibitory activity on integrin GPIIb-IIIa-dependent platelet aggregation. A vast number of disintegrins have been described since trigramin from Trimeresurus gramineus [13] and echistatin from Echis species [14] venoms were first characterised. All disintegrins contain an RGD or KGD sequence that binds with high affinity to GPIIb-IIIa and therefore competes with adhesive macromolecule ligands, capable of sustaining platelet aggregation (i.e., fibrinogen and von Willebrand factor $[15,16])$. It is interesting that, unlike fibrinogen or von Willebrand factor, venom disintegrins bind to GPIIb-IIIa without requiring prior activation of the integrin. This is because of their unique structure with the RGD sequence located at the tip of a Cys-Cys loop, facilitating binding to the receptor and expression of a ligand-induced binding site on the $\beta_{3}$ subunit of the integrin [17]. The RGDcontaining venom disintegrins inhibit platelet aggregation induced by the majority of surface receptors consistent with the central role of GPIIb-IIIa in platelet function. Disintegrins are not discussed further, and the reader 
is referred to a recent review for additional information [1].

Metalloproteinases. Venom metalloproteinases from true vipers and pit vipers are responsible for local and systemic haemorrhage due to proteolysis of the major components of the extracellular matrix [18]. They range from 20 to $100 \mathrm{kD}$, with several sizes often being found in the same venom. In general, the larger metalloproteinases are more active in inducing haemorrhage.

The complete primary structure of a high molecular weight metalloproteinase, HR1b from Trimeresurus flavoviridis venom was shown for the first time by Takeya et al. [19] in 1990. A unique structure was unravelled comprising an $\mathrm{N}$-terminus with the catalytic site, followed by a sequence with high homology to the RGD-disintegrins except that, instead of an RGD, an ECD sequence was found together with a cysteine-rich C-terminal domain. The sequences of the cDNA clones of high molecular weight metalloproteinases from Bothrops jararaca venom, jararhagin [20], and from C. atrox venom, Ht-e [21], also have a similar structural organisation (fig. 1). In addition, a pro-enzyme peptide domain at the N-terminus was found, which must be cleaved from the mature protein to allow enzyme activity. This class of metalloproteinases is named MDC (metalloproteinase/disintegrin/cysteine-rich) enzymes and shares homology to the cell membranebound family of metalloproteinases known as ADAM (a disintegrin and metalloproteinase) [22]. ADAMs are responsible for cell surface protein shedding or activation, one classical example being TACE (TNF-alpha converting enzyme) [23].

Based on their sizes, the venom metalloproteinases are classified as P-I (metalloproteinase domain only), P-II (metalloproteinase and disintegrin-like domains), P-III (MDC) and P-IV (MDC and C-type lectin domains)
[18]. Because disintegrin-like structures containing the cysteine-rich region (approximately $30 \mathrm{kD}$ ) without the metalloproteinase domain can be isolated from the whole venom, it is believed that they are products of autoproteolysis. Examples are jararhagin C [24] and catrocollastatin C [25], derived respectively from the $52-\mathrm{kD}$ jararhagin from $B$. jararaca venom and $50-\mathrm{kD}$ catrocollastatin from $C$. atrox venom [26]. Disintegrin-like components from metalloproteinases have also been produced by recombinant expression [27, 28].

\section{Snake Venom Proteins That Target}

GPIb-IX-V

GPIb-IX-V is one of the two major receptors for von Willebrand factor on the platelet surface. The second major receptor, the integrin GPIIb-IIIa, is not discussed further in this review. GPIb-IX-V is composed of two, disulphide-linked GPIb $\alpha$ and $\beta$ chains, two GPIX chains and one GPV chain. There is no similar receptor complex in the genome. The interaction of von Willebrand factor with GPIb-IX-V is indispensable for platelet adhesion to the damaged subendothelium at high shear. Von Willebrand factor promotes the initial adhesion of platelets to the exposed subendothelium due to the fast on-rate of association with GPIb-IX-V. Because of a fast off-rate of association of von Willebrand factor from the receptor complex, however, a second interaction is required for stable adhesion, such as that provided through the interaction of collagen with platelet surface receptors. Von Willebrand factor is not required for adhesion under low shear conditions.

There is also overwhelming evidence in support of a role of GPIb-IX-V in the generation of intracellular signals that lead, among other responses, to activation of GPIIb-IIIa [29]. Despite a remarkable repertoire of intracellular signals that have been reported to lie 
downstream of the GPIb-IX-V complex, the sequence of events which underlies activation of the integrin, however, remains unclear; this situation is at least partly due to the relatively weak nature of the signal from the receptor complex.

Over the years, snake venom toxins have provided valuable information on the sites of interaction of von Willebrand factor with the GPIb $\alpha$ subunit and have also been routinely used to promote the interaction of the adhesion protein with the receptor complex, an interaction that does not normally take place in the absence of high shear because of conformational constraints on von Willebrand factor. Snake venom toxins have also provided important new insights into the signalling pathways linked to the GPIb-IX-V receptor complex.

C-Type Lectins. A variety of viper venom proteins of the C-type lectin family target the platelet GPIb-IX-V complex. These venom proteins bind within the N-terminal 282 residues of GPIba [30], the same region of the receptor that contains the binding site for von Willebrand factor and other ligands such as Mac-1, P-selectin and $\alpha$-thrombin [29]. One of the reasons for the current interest in GPI$b \alpha$-targeting viper venom proteins is the extent to which these reagents mimic von Willebrand factor. In this regard, the mechanism by which von Willebrand factor binding to GPIb-IX-V induces signal transduction and platelet activation is contentious. For instance, is platelet activation that follows engagement of GPIb-IX-V by von Willebrand factor (or other ligands) initiated by crosslinking of one or more GP Ib-IX-V complexes or through the involvement of other receptors that may be associated with GPIb-IX-V? Recent studies of GPIb-IX-V-targeting venom proteins have provided fresh insights into potential functional consequences of ligand binding to this receptor.
Platelets are activated in response to binding of native multimeric von Willebrand factor or asialo-von Willebrand factor [29, 31] or recombinant von Willebrand factor A1 domain, multimerised by expression on the surface of COS cells [32]. In contrast, a monomeric, soluble fragment of von Willebrand factor encompassing the A1 domain (Glu480Gly718) binds to GPIb $\alpha$ and inhibits binding of native von Willebrand factor, but does not induce platelet aggregation [29].

The von Willebrand factor A1 domain fragment induced phosphorylation of Syk in platelets, and its association with Src, but only native multimeric von Willebrand factor induced activation of $\alpha \operatorname{IIb} \beta 3$ [33]. Like monomeric von Willebrand factor fragment, a number of approximately 25 -kD GPIb $\alpha$-binding venom proteins of the C-type lectin family also block von Willebrand factor binding, but do not activate platelets [30, 34]. The C-type lectin echicetin, from the saw-scaled viper Echis carinatus, is one such lectin which binds to GPIba and inhibits binding of both von Willebrand factor and thrombin [34, 35]. Echicitin prevents agglutination of washed platelets by von Willebrand factor [34, 35]. However, in plasma, echicetin becomes crosslinked via IgM $\kappa$, leading to agglutination, increased expression of P-selectin and activation of GPIIb-IIIa [36]. These responses can also be induced by echicetin in washed platelets by addition of IgM $\kappa$ or via cross-linking of biotinylated echicetin with avidin, and are accompanied by measurable tyrosine phosphorylation of proteins including Lyn and Syk. Thus, the major physiological action of echicitin in plasma is to promote platelet agglutination rather than inhibition, as previously thought. This may account for the thrombocytopenia seen in vivo in response to echicitin and underscores the need to test venom proteins in plasma and whole blood, where they are able to interact with other 
endogenous constituents. These results add further support to the model in which crosslinking is required for GPIb-IX-V to induce intracellular signalling events.

A higher molecular weight form of a C-type lectin-like GPIb $\alpha$-binding venom protein, 50$\mathrm{kD}$ alboaggregin A from the white lipped tree viper Trimeresurus albolabris, is also a potent platelet agonist [37-39]. However, recent reports suggest that the platelet collagen receptor, GPVI, makes a significant contribution to alboaggregin A-dependent platelet activation [40, 41]. Treatment of platelets with alboaggregin A resulted in phosphorylation of $\mathrm{Fc}$ receptor $\gamma$-chain (FcR $\gamma$-chain), Syk and phospholipase $\mathrm{C} \gamma 2$ (PLC $\gamma 2$ ), and alboaggregin Adependent platelet aggregation and tyrosine phosphorylation were inhibitable by the Src kinase family inhibitor PP1, consistent with a signalling pathway primarily involving GPVI [40]. Alboaggregin A also bound to recombinant GPVI, expressed on K562 cells. However, selectively masking GPIb $\alpha$ by anti-GPIba antibodies, inhibitory GPIb $\alpha$-binding venom proteins or proteolysis with mocarhagin partially impairs alboaggregin A-dependent platelet activation [37, 38, 40, 41]. This suggests binding to GPIb $\alpha$ may support the interaction of alboaggregin A with GPVI. The observation, however, that alboaggregin A induces aggregation of Bernard Soulier syndrome platelets demonstrates that GPIb $\alpha$ is not essential for activation of GPVI [40].

A related C-type lectin-like protein, aggretin/rhodocytin, from the Malayan pit viper Calloselasma rhodostoma has also been proposed to bind GPIba, with signalling responses dependent on an additional interaction with the collagen receptor GPIa-IIa [42]. The requirement for GPIb $\alpha$ and GPIa-IIa in the response to aggretin/rhodocytin has, however, been recently challenged, as responses are maintained in platelets deficient in these two surface glycoproteins [43]. The mecha- nism of action of aggretin/rhodocytin is discussed below.

The involvement of receptors other than GPIb-IX-V, such as GPVI or possibly GPIaIIa, complicates interpretation of signalling responses induced by GPIb $\alpha$-binding toxins. However, such mechanisms may parallel platelet stimulation by von Willebrand factor, where initial binding of von Willebrand factor to GPIb-IX-V may similarly lead to activation of additional signalling receptors. For example, there is evidence supporting a linkage of GPIb-IX-V with the low-affinity immune receptor, Fc $\gamma$ RIIa [44] and the FcR $\gamma$-chain [45]. Cross-linking of these receptors leads to tyrosine phosphorylation of the immunoreceptor tyrosine-based activation motif (ITAM) in Fc $\gamma$ RIIa and FcR $\gamma$-chain and the binding and phosphorylation of Syk, thereby initiating a signalling pathway that activates PLC $\gamma 2$ and other effector enzymes (see signalling by the collagen receptor GPVI, below). Tyrosine phosphorylation of FcR $\gamma$-chain by von Willebrand factor has been shown to contribute to platelet aggregation [45]. Interestingly, FcR $\gamma$ chain has been shown to be associated with GPIb $\alpha$ in platelets after stimulation by alboaggregin A [39], a reagent that binds GPVI in addition to GPIba (referred to above). These findings raise the intriguing possibility of a potential functional association between GPIb $\alpha$, FcR $\gamma$-chain and/or GPVI.

The family of venom C-type lectin-like proteins, therefore, includes forms which target GPIb-IX-V and inhibit ligand binding or activate platelets by mechanisms that may involve either receptor cross-linking or enlistment of other receptors, such as GPVI or possibly GPIa-IIa. Whereas alboaggregin A has the capacity to recognise more than one receptor, other C-type lectin family members, such as convulxin (see below), appear to selectively target GPVI and are not known to bind GPIba. 
Metalloproteinases. Mocarhagin is a metalloproteinase from the venom of the Mozambique spitting cobra, Naja mossambica mossambica. Originally described as a lectin, mocarhagin was affinity-purified on heparin and found to have a profound effect on platelet reactivity [46]. Mocarhagin treatment of platelets irreversibly blocked the interaction of platelet receptor glycoprotein GPIb-IX-V with its ligand, von Willebrand factor, which initiates (patho)physiological thrombus formation at high shear stress. It was subsequently shown that mocarhagin was an approximately $55-\mathrm{kD}$ metalloproteinase, and had $\mathrm{N}$ terminal sequence homology with members of the venom metalloproteinase-disintegrin family. Mocarhagin cleaved the von Willebrand factor-binding GPIb $\alpha$ subunit of the GPIb-IX-V complex as its sole detectable substrate on surface-labelled platelets [46]. The cleavage site on GPIb $\alpha$ was determined to be between residues Glu-282/Asp-283, releasing a soluble, approximately 40-kD N-terminal fragment (His1-Glu282) and producing an extracellular, sialomucin-like domain that remained membrane-associated (and disulphide-linked to GPIb $\alpha$ ).

Mocarhagin has proven a useful probe of the structure and function of GPIba. Firstly, analysis of mocarhagin-treated platelets and mocarhagin-derived fragments of purified receptor confirmed that the von Willebrand factor recognition site on GPIb-IX-V was contained within His1-Glu282 [29]. The same domain on GPIb $\alpha$ also binds other physiological ligands of GPIb-IX-V, which include Mac-1, P-selectin and $\alpha$-thrombin. Secondly, a comparison of the mocarhagin (His1Glu282), trypsin (His1-Arg293) and cathepsin G (His1-Leu275)-generated proteolytic fragments together showed that three tyrosine residues at Tyr276, Tyr278 and Tyr279 adjacent to the mocarhagin and cathepsin $G$ cleavage sites were sulphated in the native receptor [46], consistent with the sulphation state of recombinant receptor [47, 48]. Thirdly, the anionic sulphated sequence between Asp269-Glu282 was shown to mediate von Willebrand factor binding to GPIb $\alpha$ particularly in the presence of the von Willebrand factor modulator botrocetin [46-48], and the same sequence was also confirmed as a critical element in regulating thrombin binding to GPIba [46, 48, 49].

Interestingly, while GPIb $\alpha$ was the only detectable mocarhagin substrate on platelets, mocarhagin treatment of neutrophils abolished binding of $\mathrm{P}$-selectin as a result of the specific cleavage of P-selectin glycoprotein ligand-1 (PSGL-1). The mocarhagin cleavage site on PSGL-1 was determined to be within an N-terminal anionic sequence containing sulphated tyrosine residues and analogous to that found in GPIba [50]. Like in GPIba, the PSGL-1 cleavage site was also adjacent to a sialomucin-like, membrane-associated domain. It has subsequently been shown that, like PSGL-1, GPIb $\alpha$ also specifically binds Pselectin [51], and its interaction with both PSGL-1 and GPIba involves, at least in part, their sulphated tyrosine motifs [50-52]. Mocarhagin, therefore, which selectively cleaves both PSGL-1 and GPIba, potentially has both 'anti-thrombotic' and 'anti-inflammatory' activity, by specifically targeting these functionally analogous receptors involved in initial contact of circulating neutrophils (PSGL-1) or platelets (GPIb $\alpha$ ) with the blood vessel wall.

\section{Snake Venom Proteins That Target Collagen Receptors}

Subendothelial collagen fibres, being the most thrombogenic component of the vessel wall, support adhesion of platelets to the damaged subendothelium and promote activation. Collagen fibres support adhesion indirectly through von Willebrand factor, which binds to GPIb-IX-V, and directly through 
platelet surface receptors, of which the integrin GPIa-IIa $\left(\alpha_{2} \beta_{1}\right)$ and GPVI are considered to be the most important. Collagen induces platelet activation through GPVI, which is present in a complex with the FcR $\gamma$-chain. Activation of GPVI leads to tyrosine phosphorylation of FcR $\gamma$-chain on a conserved motif known as an ITAM by an Src family kinase. A second tyrosine kinase, Syk, binds to the phosphorylated ITAM, initiating a downstream signalling cascade that culminates in activation of downstream effectors including PLC $\gamma 2$, phosphatidylinositol 3-kinase and low molecular weight GTP binding proteins. The effector pathways elicit powerful platelet activation leading to GPIIb-IIIadependent aggregation, secretion and procoagulant activity. It is unclear whether the integrin GPIa-IIa also generates intracellular signals which contribute to functional responses. The interaction of platelets with collagen has been the subject of a number of recent reviews [53-56].

It is only within the last few years that snake venom toxins have begun to have a major impact on our understanding of platelet collagen interactions. The realization in 1997 that the C-type snake major venom convulxin induced platelet activation through GPVI helped to bring this receptor to the forefront of the platelet-collagen field and was pivotal in the eventual cloning of the glycoprotein. Several snake venom toxins are now recognised to target GPVI, and it seems likely that many more will be found in the coming years.

\section{Snake Venom Toxins Acting on GPVI}

C-Type Lectin. Convulxin is a C-type lectin from the venom of the South American tropical rattlesnake Crotalus durissus terrificus. Convulxin was cloned in 1998 [7]. It consists of two subunits, $\alpha$ and $\beta$, joined by disulphide bridges in a trimer-trimer organisation.
Convulxin was initially described in 1970 [57] and shown to be a powerful platelet agonist in 1980 [58]. Within 2 years of this report, convulxin was proposed to activate platelets through a pathway that was also used by collagen on the basis of cross-desensitisation between the two stimuli [59]. However, it was a further 15 years before it was realised to mediate activation through the major activatory collagen receptor, GPVI $[9,60]$. Several lines of evidence were presented in support of this conclusion including: (1) the ability of a polyclonal antiserum to GPVI to block platelet activation by convulxin; (2) the ability of lower molecular weight fragments of convulxin to block platelet activation by collagen; (3) the similar pattern of signal transduction events mediated by convulxin and collagen including marked tyrosine phosphorylation of FcR $\gamma$ chain, Syk and PLC $\gamma 2$, and (4) a ligand blot assay demonstrated that convulxin bound to a protein of $62 \mathrm{kD}$ which comigrated with GPVI under non-reducing conditions [60, 61]. The activity of convulxin on GPVI has since been confirmed on cells transfected with the glycoprotein receptor [62].

There are also reports that the action of convulxin is partially inhibited by antibodies to GPIa-IIa, raising the possibility that the integrin is, in some unknown way, linked to the action of GPVI in platelets [9,63]. This observation should be treated with caution, however, in that antibodies to the integrin also have weak inhibitory effects on platelet activation by other agonists which signal independence of the integrin including thrombin [64] and aggretin/rhodocytin (see 'The Mechanism of Action of Aggretin/Rhodocytin'). The observation that iodinated-convulxin binds to one [65] or possibly two sites on the platelet surface [9] should also be treated with caution, bearing in mind the multisubunit composition of the toxin and that full iodination destroys activity. The two binding 
sites might reflect heterogeneity within the iodinated-convulxin causing affinity differences. Interestingly, collagen-related peptides, which are specific GPVI, only partially (approximately $30 \%$ ) inhibit the binding of convulxin demonstrating binding to distinct sites on GPVI [66].

Convulxin has subnanomolar affinity for GPVI and, therefore, functions as a pseudoirreversible ligand [65]. This makes it an ideal reagent as an affinity ligand, a property that was exploited by Clemetson et al. [62] in 1999 in the cloning of GPVI. GPVI is a novel member of the Ig family of surface glycoproteins with strong sequence homology to the $F c \alpha$ receptor and natural killer inhibitory receptors. GPVI has recently been extensively reviewed by Clemetson et al. [55].

Convulxin is now widely used as a specific agonist in the dissection of GPVI-regulated signal transduction events because of its selective action on GPVI [67, 68]. However, it should be borne in mind that convulxin is a much more powerful agonist than collagen and, therefore, much less reliant on release of secondary mediators to bring about platelet activation [69]. Convulxin is, therefore, not an accurate mimetic of collagen-based platelet interactions.

A number of other C-type lectins which mediate their actions through GPVI have been described. Trimucytin from the venom of Trimeresurus mucrosquamatus was originally described as a ligand for GPIa-IIa on account of the weak inhibitory action of the GPIa-IIa mAb 6F1 against platelet activation [70]. However, Hers et al. [64] reported a similar weak inhibitory effect of $\mathrm{mAb} 6 \mathrm{~F} 1$ against a number of other platelet agonists and further demonstrated that the monoclonal antibody was unable to block responses to higher concentrations of the snake venom toxin. Moreover, in common with other GPVIselective agonists, trimucytin stimulated marked tyrosine phosphorylation of the FcR $\gamma$-chain, Syk and PLC $\gamma 2$, suggesting it may be an agonist at GPVI [64]. This hypothesis requires confirmation of the activity of trimucytin on GPVI-transfected cells. It is also possible that trimucytin binds to both GPVI and GPIa-IIa.

A further example of a C-type lectin that binds to GPVI is alboaggregin A which, as discussed above, also binds to GPIba [40, 41]. A second C-type lectin with dual affinity for GPVI and GPIba, alboluxin, has also been identified in the venom from Trimeresurus albolabris [71]. It is interesting to speculate whether activity at more than one platelet surface glycoprotein will be common to many venom proteins.

Metalloproteinases. The $60-\mathrm{kD}$ metalloproteinase-disintegrin alborhagin from the venom of the white-lipped tree viper Trimeresurus albolabris has recently been shown to be a potent agonist at GPVI, demonstrating the versatility of snake venom toxins of distinct structures in targeting a common substrate [72]. Interestingly, alborhagin was isolated from the same venom as for alboaggregin A, demonstrating that a single venom can contain proteins of distinct structural classes with common functions.

The evidence demonstrating that alborhagin mediates its activation through GPVI includes the similar pattern of protein tyrosine phosphorylation to that induced by convulxin in platelets, including tyrosine phosphorylation of PLC 22 and the ability of the toxin to activate GPVI expressed in Jurkat $\mathrm{T}$ cells and K562 cells [72]. Alborhagin was unable to displace the binding of convulxin to GPVI in platelets or in GPVI-transfected cells, using a flow cytometry-based assay demonstrating that the two toxins bind to the glycoprotein receptor at distinct sites. On the other hand, the rat anti-GPVI monoclonal antibody, JAQ1, which has been previously 
shown to block platelet activation by collagenrelated peptides [73], also blocked activation by alborhagin, suggesting a common site of interaction on the glycoprotein receptor. This makes alborhagin a novel probe to study the function of GPVI.

\section{Snake Venom Toxins Acting on GPIa-IIa} ( $\alpha_{2} \beta_{1}$ Integrin)

C-Type Lectin. The snake C-type lectins trimucytin and aggretin/rhodocytin were originally proposed to interact with GPIa-IIa [70, 74], although this has been brought into question as discussed in 'C-type lectins' and 'The Mechanism of Action of Aggretin/Rhodocytin'. There is therefore no definitive evidence, at present, for a role of GPIa-IIa in the action of platelet-activatory snake venom toxins. Whether this reflects a minimal role of the integrin in the generation of intracellular signalling events in platelets is a subject for speculation. On the other hand, an inhibitory Ctype lectin whose binding prevents binding of collagen to GPIa-IIa has been described [75, 76]. Rhodocetin is a venom component of the Malayan pit viper Calloselasma rhodostoma, which is also the source of aggretin/rhodocytin. Rhodocetin is a heterodimer of two subunits of 16 and $14 \mathrm{kD}$. Neither subunit has a collagenous triple helical domain or an RGDbinding sequence, and binding to the integrin is independent of divalent cations. The structural basis of the way in which rhodocetin binds to GPIa-IIa is, therefore, of considerable interest as this may pave the way for development of non-helical ligands for the integrin.

It is interesting to consider why a single snake venom might express a toxin which stimulates platelet activation and one that inhibits this response, although this is not in itself an unusual finding. In the present case, it is possible that the major role of rhodocytin is to target GPIa-IIa in tissues other than the platelet that express GPIa-IIa, such as endothelial cells and fibroblasts. Interestingly, the venom of the Malayan pit viper also contains a second GPIa-IIa inhibiting activity of high molecular weight which has yet to be characterised [76].

Metalloproteinases. The class P-III metalloproteinases, jararhagin and catrocollastatin, inhibit platelet adhesion to collagen [25, 77]. Whilst this class of enzymes is best known for its ability to degrade extracellular matrix proteins, it does not use type I or III collagen as substrate [18]. With catrocollastatin, Zhou et al. [25] reported that the mechanism of inhibition of platelet aggregation was through the direct binding of catrocollastatin to collagen. Jararhagin, on the other hand, appears to cleave the integrin GPIa-IIa [78]. GPVI is not affected by jararhagin, as convulxin binding to GPVI and the subsequent downstream signalling events are unaltered in jararhagintreated platelets [63]. Jararhagin cleaves the GPIIa $\left(\beta_{1}\right)$ subunit of the integrin receptor, which remains associated by a disulphide bond to the receptor complex [77, 78]. As a consequence of this, the function of the receptor for aggregation or recognition by antibodies targeting the functional I domain of GPIa subunit $\left(\alpha_{2}\right)$, responsible for the recognition of native collagen, is lost.

To bring the enzyme close to the platelet GPIa-IIa, it is crucial that jararhagin binds to platelets. However, the binding of the active enzyme to platelets is weak and can only be demonstrated after enzyme treatment with 1,10-phenanthroline, a general inhibitor of metalloproteinases [77]. It is, however, possible that a transient binding of the active enzyme takes place but that, following cleavage, jararhagin does not remain bound to the receptor. Similarly to integrin recognition by the RGD disintegrins, it is then logical to assume that the disintegrin-like domain of jararhagin also plays a role in the binding. 
Table 1. Snake venom toxins that target platelet vWf and collagen and their receptors GPIb-IX-V, GPVI and GPIa-IIa

\begin{tabular}{|c|c|c|c|c|}
\hline \multicolumn{3}{|c|}{ Venom protein family } & \multirow[t]{2}{*}{ Function } & \multirow[t]{2}{*}{ References } \\
\hline target & protein & snake species & & \\
\hline \multicolumn{5}{|c|}{ C-type lectin-like family } \\
\hline \multirow[t]{3}{*}{ vWf } & aspercetin & Bothrops asper & binding to vWF causing agglutination & 84 \\
\hline & bitiscetin & Bitis arietans & binding to vWF causing agglutination & 85 \\
\hline & botrocetin & Bothrops jararaca & binding to $\mathrm{vWF}$ causing agglutination & 8 \\
\hline \multirow[t]{9}{*}{ GPIb $\alpha$, GPVI } & agkicetin & Agkistrodon acutus & blocks vWf & 86 \\
\hline & alboaggregin $\mathrm{B}$ & Trimeresurus albolabris & blocks vWf, agglutinates platelets & $37,87,88$ \\
\hline & $\mathrm{CHH}-\mathrm{A}$ and $-\mathrm{B}$ & Crotalus horridus horridus & block vWf & 37 \\
\hline & crotalin & Crotalus atrox & blocks vWf & 89 \\
\hline & echicetin & Echis carinatus & blocks vWf (and $\alpha$-thrombin) & 35 \\
\hline & flavocetin A and B & Trimeresurus flavoviridus & block & \\
\hline & mamushigin & Agkistrodon halys blomhoffi & $\begin{array}{l}\text { blocks vWf binding and aggregates } \\
\text { platelets under shear }\end{array}$ & 90,91 \\
\hline & jararaca GPIb-BP & Bothrops jararaca & blocks vWf & 92 \\
\hline & tokaracetin & Trimeresurus tokarensis & blocks vWf & 93 \\
\hline GPIb $\alpha$, GPVI & alboaggregin $\mathrm{A}$ & Trimeresurus albolabris & activates platelets & $37,38,40,41$ \\
\hline \multirow[t]{2}{*}{ GPVI } & convulxin & Crotalus durissus terrificus & activates platelets & 9,60 \\
\hline & trimucytin & Trimeresurus mucrosquamatus & activates platelets & 70 \\
\hline GPIa-IIa & rhodocetin & Calloselasma rhodostoma & inhibits binding of collagen & 76 \\
\hline \multicolumn{5}{|c|}{ Metalloproteinase-disintegrin family } \\
\hline Collagen & catrocollastatin & Crotalus atrox & $\begin{array}{l}\text { binds to collagen, inhibiting } \\
\text { aggregation }\end{array}$ & 25 \\
\hline vWf & kauothiagin & Naja kaouthia & $\begin{array}{l}\text { cleaves vWf leading to loss of binding } \\
\text { to collagen and ristocetin-aggregation }\end{array}$ & 94 \\
\hline GPVI & alborhagin & Trimeresurus albolabris & activates platelets & 72 \\
\hline GPIb $\alpha$, GPVI & mocarhagin & Naja mossambica mossambica & $\begin{array}{l}\text { cleaves GPIb } \alpha \text { (Glu282/Asp283), } \\
\text { abolishes vWf binding }\end{array}$ & 46 \\
\hline GPIa-IIa & jararhagin & Bothrops jararaca & $\begin{array}{l}\text { cleaves GPIIa, abolishes collagen- } \\
\text { induced platelet aggregation }\end{array}$ & 77 \\
\hline
\end{tabular}

vWF = von Willebrand factor.

Indeed, it has been shown that jararhagin C, the proteolytic product of jararhagin, inhibits collagen-induced platelet aggregation [24, 27].

With regard to determining the jararhagin interacting site with GPIa/IIa, synthetic peptides containing the sequence RKKH of the metalloproteinase domain of jararhagin have been shown to bind to the I domain of the GPIa $\left(\alpha_{2}\right)$ subunit of GPIa/IIa [79, 80]. This sequence also inhibits platelet aggregation by collagen [Kamiguti and Fox, unpubl.]. How- ever, synthetic peptides based on the disintegrin-like region of either jararhagin [78] or atrolysin A [28] are also inhibitors, indicating a multidomain function in platelet/collagen interaction by these enzymes. Additional studies are required to fully understand these events, although it is clear that the mechanism through which jararhagin inhibits collageninduced platelet aggregation involves binding to GPIa and cleavage of the GPIIa subunit. 


\section{Snake Venom Toxins Acting on Other}

Collagen Receptors

A number of other receptors have been proposed for collagen on the platelet surface including CD36 (GPIV) and p65. The role of these proteins in the physiological function of collagen, however, remains unclear. In this context, it is notable that no snake venom toxins have been reported which mediate their actions through these putative collagen receptors.

\section{The Mechanism of Action of \\ Aggretin/Rhodocytin}

As discussed above, the C-type lectin aggretin from C. rhodostoma was originally proposed to activate platelets through the collagen receptor integrin GPIa-IIa based on the inhibitory effect of antibodies to the integrin [74]. Subsequently, Shin and Morita [81] isolated a C-type lectin from the same snake venom which also activated platelets independently of GPIb $\alpha$, but whose action was blocked by antibodies to GPIa-IIa [82, 83]. Aggretin and rhodocytin share the same Nterminal amino sequences and are present in a tetrameric structure. However, they do have a number of different problems, making it unclear whether they are the same protein or closely related variants.

Aggretin/rhodocytin have been shown to activate platelets through a tyrosine kinasedependent pathway that has a number of similarities to the cascade induced by the collagen receptor GPVI including tyrosine phosphorylation of Syk and PLC 22 [42, 83]. However, it is clear that the toxin does not induce activation through this receptor, since the response is maintained in FcR $\gamma$-chain-deficient platelets, which lack the glycoprotein receptor [42, 83]. Furthermore, tyrosine phosphorylation of Syk and PLC $\gamma 2$ was mediated through a cyclooxygenase-sensitive pathway, in contrast to the response to the collagen receptor GPVI
[83]. Navdaev et al. [42] have proposed that aggretin/rhodocytin activates platelets through cross-linking of GPIb $\alpha$ and GPIa-IIa, based on the observation that some but not all antibodies to these surface glycoproteins block platelet activation by the toxin. This conclusion however has been brought into question by the observations that (1) aggretin/ rhodocytin does not bind to recombinant GPIa-IIa [76] and (2) the toxin activates GPIa-IIa-deficient murine platelets in which GPIb $\alpha$ has also been removed through treatment with mocarhagin [43]. These observations provide unequivocal evidence that the action of aggretin/rhodocytin is mediated at least partly through a novel surface receptor. The identification of this surface receptor is an area of considerable interest.

Table 1 provides a summary of snake venom toxins that target platelet von Willebrand factor and collagen and their receptors GPIbIX-V, GPVI and GPIa-IIa.

\section{Concluding Remarks}

This review has focused on the action of snake venom toxins, on the actions of two of the major platelet-adhesive ligands: von Willebrand factor and collagen. The contribution that snake venom toxins have made to our understanding of the way these two adhesive ligands interact with platelet receptors has been, and continues to be, immense. In addition, snake venom toxins have made substantial contributions to our understanding of the role of the integrin GPIIb-IIIa in platelet function and in the action of other platelet agonists and their receptors. Given this background, it is remarkable that, for example, it took almost 20 years to identify GPVI as the receptor for convulxin and to exploit the use of the toxin in the eventual purification and cloning of the glycoprotein. 
It is interesting to speculate whether snake venom toxins will continue to provide new and novel tools to study receptors for von Willebrand factor and collagen, as well as other platelet ligands. This seems highly likely, bearing in mind that three novel ligands have been described for GPVI in the past 12 months. Moreover, given the large number of venomous snakes in the world and that many venoms have not been rigorously tested, it seems inevitable that new types of activities will be discovered over the coming years, possibly leading to the identification of further, novel activatory or inhibitory platelet recep- tors, or new tools with which to dissect the function of receptors for adhesive ligands. In this context, rhodocytin/aggretin represents a particularly promising example of a snake venom toxin that appears to mediate at least part of its action through a novel pathway linked to a previously unidentified platelet receptor.

\section{Acknowledgements}

S.P.W. thanks the Welcome Trust and British Heart Foundation for support. S.P.W. is a BHF Senior Research Fellow.

\section{References}

1 Markland FS: Snake venoms and the hemostatic system. Toxicon 1998;36:1749-1800.

2 Drickamer K: Two distinct classes of carbohydrate-recognition domains in animal lectins. J Biol Chem 1988;263:9557-9560.

3 Spiess M: The asialoglycoprotein receptor: A model for endocytic transport receptors. Biochemistry 1990; 29:10009-10018.

4 Weis WI, Crichlow GV, Murthy HM, Hendrickson WA, Drickamer $\mathrm{K}$ : Physical characterization and crystallization of the carbohydraterecognition domain of a mannosebinding protein from rat. J Biol Chem 1991;266:20678-20686.

5 Weis WI, Drickamer K, Hendrickson WA: Structure of a C-type mannose-binding protein complexed with an oligosaccharide. Nature 1992;360:127-134.

6 Hirabayashi J: Two distinct families of animal lectins: Speculation on their raison d'être; in van Driessche E, Fischer J, Beeckmans S, BøgHansen TC (eds): Lectins. Hellerup, Textop, 1993, vol 10, pp 205-219.

7 Leduc M, Bon C: Cloning of subunits of convulxin, a collagen-like platelet-aggregating protein from Crotalus durissus terrificus venom. Biochem J 1998;333:389-393.
8 Usami Y, Fujimura Y, Suzuki M, Ozeki Y, Nishio K, Fukui H, Titani $\mathrm{K}$ : Primary structure of two-chain botrocetin, a von Willebrand factor modulator purified from the venom of Bothrops jararaca. Proc Natl Acad Sci USA 1993;90:928-932.

9 Jandrot-Perrus M, Lagrue AH, Okuma M, Bon C: Adhesion and activation of human platelets induced by convulxin involve glycoprotein VI and integrin alpha2beta1. J Biol Chem 1997;272:27035-27041.

10 Mizuno H, Fujimoto Z, Koizumi M, Kano H, Atoda H, Morita T: Structure of coagulation factors IX/Xbinding protein, a heterodimer of $C$ type lectin domains. Nat Struct Biol 1997;4:438-441.

11 Mizuno H, Fujimoto Z, Koizumi M, Kano H, Atoda H, Morita T: Crystal structure of coagulation factor IXbinding protein from habu snake venom at 2.6 A: Implication of central loop swapping based on deletion in the linker region. J Mol Biol 1999; 289:103-112.

12 Sen U, Vasudevan S, Subbarao G, McClintock RA, Celikel R, Ruggeri ZM, Varughese KI: Crystal structure of the von Willebrand factor modulator botrocetin. Biochemistry 2001;40:345-352.
13 Huang TF, Holt JC, Lukasiewicz H, Niewiarowski S: Trigramin. A low molecular weight peptide inhibiting fibrinogen interaction with platelet receptors expressed on glycoprotein IIb-IIIa complex. J Biol Chem 1987; 262:16157-16163.

14 Gan ZR, Gould RJ, Jacobs JW, Friedman PA, Polokoff MA: Echistatin. A potent platelet aggregation inhibitor from the venom of the viper, Echis carinatus. J Biol Chem 1988;263:19827-19832.

15 Scarborough RM, Rose JW, Naughton MA, Phillips DR, Nannizzi L, Arfsten A, Campbell AM Charo IF: Characterization of the integrin specificities of disintegrins isolated from American pit viper venoms. J Biol Chem 1993;268:1058-1065.

16 Niewiarowski S, McLane MA, Kloczewiak M, Stewart GJ: Disintegrins and other naturally occurring antagonists of platelet fibrinogen receptors. Semin Hematol 1994;31:289300.

17 Juliano D, Wang Y, Marcinkiewicz C, Rosenthal LA, Stewart GJ, Niewiarowski S: Disintegrin interaction with alpha $\mathrm{V}$ beta 3 integrin on human umbilical vein endothelial cells: Expression of ligand-induced binding site on beta 3 subunit. Exp Cell Res 1996;225:132-142. 
18 Bjarnason JB, Fox JW: Hemorrhagic metalloproteinases from snake venoms. Pharmacol Ther 1994;62: 325-372.

19 Takeya H, Oda K, Miyata T, OmoriSatoh T, Iwanaga S: The complete amino acid sequence of the high molecular mass hemorrhagic protein HR1B isolated from the venom of Trimeresurus flavoviridis. J Biol Chem 1990;265:16068-16073.

20 Paine MJ, Desmond HP, Theakston RD, Crampton JM: Purification, cloning, and molecular characterization of a high molecular weight hemorrhagic metalloprotease, jararhagin, from Bothrops jararaca venom. Insights into the disintegrin gene family. J Biol Chem 1992;267: 22869-22876.

21 Hite LA, Shannon JD, Bjarnason JB, Fox JW: Sequence of a cDNA clone encoding the zinc metalloproteinase hemorrhagic toxin e from Crotalus atrox: Evidence for signal, zymogen, and disintegrin-like structures. Biochemistry 1992;31:62036211.

22 Wolfsberg TG, Straight PD, Gerena RL, Huovila AP, Primakoff P, Myles DG, White JM: ADAM, a widely distributed and developmentally regulated gene family encoding membrane proteins with a disintegrin and metalloprotease domain. Dev Biol 1995;169:378-383.

23 Black RA Rauch CT, Kozlosky CJ, Peschon JJ, Slack JL, Wolfson MF, Castner BJ, Stocking KL, Reddy P, Srinivasan S, Nelson N, Boiani N, Schooley KA, Gerhart M, Davis R, Fitzner JN, Johnson RS, Paxton RJ, March CJ, Cerretti DP: A metalloproteinase disintegrin that releases tumour-necrosis factor-alpha from cells. Nature 1997;385:729-733.

24 Usami Y, Fujimura Y, Miura S, Shima H, Yoshida E, Yoshioka A, Hirano K, Suzuki M, Titani K: A 28 kDa-protein with disintegrin-like structure (jararhagin-C) purified from Bothrops jararaca venom inhibits collagen- and ADP-induced platelet aggregation. Biochem Biophys Res Commun 1994;201:331339.
25 Zhou Q, Dangelmaier C, Smith JB: The hemorrhagin catrocollastatin inhibits collagen-induced platelet aggregation by binding to collagen via its disintegrin-like domain. Biochem Biophys Res Commun 1996; 219:720-726.

26 Smith JB, Dangelmaier C, Selak M: Identification of $50 \mathrm{kDa}$ snake venom proteins which specifically inhibit platelet adhesion to collagen. FEBS Lett 1991;283:307-310.

27 Moura-da-Silva AM, Linica A, Della-Casa MS, Kamiguti AS, Ho PL, Crampton JM, Theakston RD: Jararhagin ECD-containing disintegrin domain: Expression in Escherichia coli and inhibition of the platelet-collagen interaction. Arch Biochem Biophys 1999;369:295-301.

28 Jia LG, Wang XM, Shannon JD, Bjarnason JB, Fox JW: Inhibition of platelet aggregation by the recombinant cysteine-rich domain of the hemorrhagic snake venom metalloproteinase, atrolysin A. Arch Biochem Biophys 2000;373:281-286.

29 Berndt MC, Shen Y, Dopheide SM, Gardiner EE, Andrews RK: The vascular biology of the glycoprotein Ib-IX-V complex. Thromb Haemost 2001;86:178-189.

30 Andrews RK, Berndt MC: Snake venom modulators of platelet adhesion receptors and their ligands. Toxicon 2000;38:775-791.

31 Kulkarni S, Dopheide SM, Yap CL, Ravanat C, Freund M, Mangin P, Heel KA, Street A, Harper IS, Lanza F, Jackson SP: A revised model of platelet aggregation. J Clin Invest 2000; 105:783-791.

32 Schulte am Esch J 2nd, Cruz MA, Siegel JB, Anrather J, Robson SC: Activation of human platelets by the membrane-expressed A1 domain of von Willebrand factor. Blood 1997; 90:4425-4437.

33 Satoh K, Asazuma N, Yatomi Y, Fujimura Y, Miura S, Titani K, Ozaki Y: Activation of protein-tyrosine kinase pathways in human platelets stimulated with the A1 domain of von Willebrand factor. Platelets 2000;11:171-176.

34 Fujimura Y, Kawasaki T, Titani K: Snake venom proteins modulating the interaction between von Willebrand factor and platelet glycoprotein Ib. Thromb Haemost 1996;76: 633-639.
35 Peng M, Lu W, Beviglia L, Niewiarowski S, Kirby EP: Echicetin: A snake venom protein that inhibits binding of von Willebrand factor and alboaggregins to platelet glycoprotein Ib. Blood 1993;81:23212328.

36 Navdaev A, Dormann D, Clemetson JM, Clemetson KJ: Echicetin, a GPIb-binding snake C-type lectin from Echis carinatus, also contains a binding site for IgMkappa responsible for platelet agglutination in plasma and inducing signal transduction. Blood 2001;97:2333-2341.

37 Andrews RK, Kroll MH, Ward CM, Rose JW, Scarborough RM, Smith AI, Lopez JA, Berndt MC: Binding of a novel 50-kilodalton alboaggregin from Trimeresurus albolabris and related viper venom proteins to the platelet membrane glycoprotein Ib-IX-V complex. Effect on platelet aggregation and glycoprotein Ib-mediated platelet activation. Biochemistry 1996;35:12629-12639.

38 Kowalska MA, Tan L, Holt JC, Peng M, Karczewski J, Calvete JJ, Niewiarowski S: Alboaggregins A and B. Structure and interaction with human platelets. Thromb Haemost 1998;79:609-613.

39 Falati S, Edmead CE, Poole AW: Glycoprotein Ib-V-IX, a receptor for von Willebrand factor, couples physically and functionally to the $\mathrm{Fc}$ receptor gamma-chain, Fyn, and Lyn to activate human platelets. Blood 1999;94:1648-1656.

40 Asazuma N, Marshall SJ, Berlanga O, Snell D, Poole AW, Berndt MC, Andrews RK, Watson SP: The snake venom toxin alboaggregin-A activates glycoprotein VI. Blood 2001; 97:3989-3991.

41 Dormann D, Clemetson JM, Navdaev A, Kehrel BE, Clemetson KJ: Alboaggregin A activates platelets by a mechanism involving glycoprotein VI as well as glycoprotein Ib. Blood 2001;97:929-936. 
42 Navdaev A, Clemetson JM, Polgar J, Kehrel BE, Glauner M, Magnenat E, Wells TN, Clemetson KJ: Aggretin, a heterodimeric C-type lectin from Calloselasma rhodostoma (Malayan pit viper) stimulates platelets by binding to alpha 2 beta 1 integrin and glycoprotein $\mathrm{Ib}$, activating Syk and phospholipase Cgamma 2, but does not involve the glycoprotein $\mathrm{VI} / \mathrm{Fc}$ receptor gamma chain collagen receptor. J Biol Chem 2001;276:20882-20889.

43 Bergmeier W, Bouvard D, Eble JA, Mokhtari-Nejad R, Schulte V, Zirngibl H, Brakebusch C, Fässler R, Nieswandt B: Rhodocytin (aggretin) activates platelets lacking alpha(2)beta(1) integrin, glycoprotein VI, and the ligand-binding domain of glycoprotein Ibalpha. $\mathrm{J}$ Biol Chem 2001;276:25121-25126.

44 Sullam PM, Hyun WC, Szollosi J, Dong J, Foss WM, Lopez JA: Physical proximity and functional interplay of the glycoprotein Ib-IX-V complex and the Fc receptor FcgammaRIIA on the platelet plasma membrane. J Biol Chem 1998;273: 5331-5336.

45 Yi W, Suzuki-Inoue K, Satoh K, Asazuma N, Yatomi Y, Berndt MC, Ozaki Y: Role of Fc receptor-chain in platelet glycoprotein Ib-mediated signaling. Blood 2001;97:38363845.

46 Ward CM, Andrews RK, Smith AI, Berndt MC: Mocarhagin, a novel cobra venom metalloproteinase, cleaves the platelet von Willebrand factor receptor glycoprotein Ibalpha. Identification of the sulfated tyrosine/anionic sequence Tyr-276Glu-282 of glycoprotein Ibalpha as a binding site for von Willebrand factor and alpha-thrombin. Biochemistry 1996;35:4929-4238.

47 Dong JF, Li CQ, Lopez JA: Tyrosine sulfation of the glycoprotein Ib-IX complex: Identification of sulfated residues and effect on ligand binding. Biochemistry 1994;33:1394613953.

48 Marchese P, Murata M, Mazzucato M, Pradella P, De Marco L, Ware J, Ruggeri ZM: Identification of three tyrosine residues of glycoprotein $\mathrm{Ib}$ alpha with distinct roles in von Willebrand factor and alpha-thrombin binding. J Biol Chem 1995;270: 9571-9578.
49 de Cristofaro R, de Candia E, Rutella S, Weitz JI: The Asp(272)Glu(282) region of platelet glycoprotein Ibalpha interacts with the heparin-binding site of alpha-thrombin and protects the enzyme from the heparin-catalyzed inhibition by antithrombin III. J Biol Chem 2000; 275:3887-3895

50 de Luca M, Dunlop LC, Andrews RK, Flannery JV Jr, Ettling R, Cumming DA, Veldman GM, Berndt MC: A novel cobra venom metalloproteinase, mocarhagin, cleaves a 10 -amino acid peptide from the mature $\mathrm{N}$ terminus of $\mathrm{P}$-selectin glycoprotein ligand receptor, PSGL-1, and abolishes P-selectin binding. J Biol Chem 1995;270:26734-26737.

51 Romo GM, Dong JF, Schade AJ, Gardiner EE, Kansas GS, Li CQ, McIntire LV, Berndt MC, Lopez JA: The glycoprotein Ib-IX-V complex is a platelet counterreceptor for Pselectin. J Exp Med 1999;190:803814.

52 Pouyani T, Seed B: PSGL-1 recognition of P-selectin is controlled by a tyrosine sulfation consensus at the PSGL-1 amino terminus. Cell 1995; 83:333-343.

53 Watson SP, Berlanga O, Best D, Frampton J: Update on collagen receptors: Is the two-site, two-state model still valid? Platelets 2000;11: 252-259.

54 Watson SP, Asazuma N, Atkinson B, Berlanga O, Best D, Bobe R, Jarvis G, Marshall S, Snell D, Stafford M, Tulasne D, Wilde J, Wonerow P, Frampton J: The role of ITAM- and ITIM-coupled receptors in platelet activation by collagen. Thromb Haemost 2001;86:276-289.

55 Clemetson K, Clemetson JM: Platelet collagen receptors. Thromb Haemost 2001;86:189-198.

56 Watson SP, Gibbins J: Collagen receptor in platelets: Extending the role of the ITAM. Immunol Today 1998;19:260-265.

57 Prado-Franceschi J: Snake Venoms in the Study of Platelet Function. Thesis, University of Campinas, 1970.
58 Vargaftig BB, Prado-Franceschi J, Chignard M, Lefort J, Marlas G: Activation of guinea-pig platelets induced by convulxin, a substance extracted from the venom of Crotalus durissus cascavella. Eur J Pharmacol 1980;68:451-458.

59 Vargaftig BB, Joseph D, Wal F, Marlas G, Chignard M, Chevance LG: Convulxin-induced activation of intact and of thrombin-degranulated rabbit platelets: Specific crossed desensitisation with collagen. Eur J Pharmacol 1982;92:5768.

60 Polgar J, Clemetson JM, Kehrel BE, Wiedemann M, Magnenat EM, Well TN, Clemetson KJ: Platelet activation and signal transduction by convulxin, a C-type lectin from Crotalus durissus terrificus (tropical rattlesnake) venom via the p62/GPVI collagen receptor. J Biol Chem 1997; 272:13576-13583

61 Francischetti IM, Ghazaleh FA, Reis RA, Carlini CR, Guinmaraes JA: Convulxin induces platelet activation by a tyrosine-kinase dependent pathway and stimulates tyrosine phosphorylation of platelet proteins, including PLC $\gamma 2$, independently of integrin $\alpha \mathrm{IIb} \beta 3$. Arch Biochem Biophys 1998;353:239-250.

62 Clemetson JM, Polgar J, Magnenat E, Wells TN, Clemetson KJ: The platelet collagen receptor glycoprotein VI is a member of the immunoglobulin superfamily closely related to Fc $\alpha \mathrm{R}$ and the natural killer receptors. J Biol Chem 1999;274:2901929024.

63 Kamiguti AS, Theakston RD, Watson SP, Bon C, Laing GD, Zuzel M: Distinct contributions of glycoprotein VI and alpha(2)beta(1) integrin to the induction of platelet protein tyrosine phosphorylation and aggregation. Arch Biochem Biophys 2000;374:356-362.

64 Hers I, Berlanga O, Tiekstra MJ, Kamiguti AS, Theakston RD, Watson SP: Evidence against a direct role of the integrin alpha2beta1 in collagen-induced tyrosine phosphorylation in human platelets. Eur J Biochem 2000;267:2088-2097. 
65 Francischetti IM, Saliou B, Leduc M, Carlini CR, Hatmi M, Randon J, Faili A, Bon C: Convulxin, a potent platelet-aggregating protein from Crotalus durissus terrificus venom, specifically binds to platelets. Toxicon 1997;35:1217-1228.

66 Niedergang F, Alcover A, Knight CG, Farndale RW, Barnes MJ, Francischetti IM, Bon C, Leduc M: Convulxin binding to platelet receptor GPVI: Competition with collagen related peptides. Biochem Biophys Res Commun 2000;273:246250.

67 Asazuma N, Wilde JI, Berlanga O, Leduc M, Leo A, Schweighoffer E, Tybulewicz V, Bon C, Liu SK, McGlade CJ, Schraven B, Watson SP: Interaction of linker for activation of T cells with multiple adapter proteins in platelets activated by the glycoprotein VI-selective ligand, convulxin. J Biol Chem 2000;275: 33427-33434.

68 Cicmil M, Thomas JM, Sage T, Barry $\mathrm{FA}$, Leduc $\mathrm{M}$, Bon $\mathrm{C}$, Gibbins JM: Collagen, convulxin, and thrombin stimulate aggregation-independent tyrosine phosphorylation of CD31 in platelets: Evidence for the involvement of Src family kinases. J Biol Chem 2000;275: 27339-27347.

69 Atkinson B, Stafford M, Pears C, Watson SP: Events underlying platelet aggregation to the GPVI agonist convulxin. Eur J Biochem 2001, in press.

70 Teng CM, Ko FN, Tsai IH, Hung ML, Huang TF: Trimucytin: A collagen-like aggregating inducer isolated from Trimeresurus mисrosquamatus snake venom. Thromb Haemost 1993;69:286-292.

71 Du XY, Magnenat E, Wells KJ, Clemetson KJ: Alboluxin, a snake C-type lectin from Trimeresurus albolabris venom is a potent platelet agonist acting via both GPIb and GPVI (abstract). Thromb Haemost 2001;86 (Jul suppl): 1160.

72 Andrews RK, Gardiner EE, Asazuma N, Berlanga O, Tulasne D, Nieswandtl B, Smith AI, Berndt MC, Watson SP: A novel viper venom metalloproteinase, alborhagin, is an agonist at the platelet collagen receptor GPVI. J Biol Chem2001;276: 28092-20897.
73 Schulte V, Snell D, Bermeier W, Zirngibl H, Watson SP, Nieswandt $\mathrm{B}$ : Evidence for two distinct epitopes within collagen for activation of murine platelets. J Biol Chem 2001;276:364-368.

74 Huang TF, Liu CZ, Yang SH: Aggretin, a novel platelet-aggregation inducer from snake (Calloselasma rhodostoma) venom, activates phospholipase $\mathrm{C}$ by acting as a glycoprotein Ia/IIa agonist. Biochem J 1995; 309:1021-1027.

75 Wang R, Kini RM, Chung MCM: Rhodocetin, a novel platelet aggregation inhibitor from the venom of Calloselasma rhodostomai (Malayan pit viper): Synergistic and noncovalent interaction between its subunits. Biochemistry 1999;38:75847593.

76 Eble JA, Beermann B, Hinz JH, Schmidt-Hederich A: Alpha2beta1 integrin is not recognized by rhodocytin but is the specific, high affinity target of rhodocetin, an RGD-independent disintegrin and potent inhibitor of cell adhesion to collagen. $\mathrm{J}$ Biol Chem 2001;276:12274-12284.

77 Kamiguti AS, Hay CR, Zuzel $\mathrm{M}$ : Inhibition of collagen-induced platelet aggregation as the result of cleavage of alpha 2 beta 1-integrin by the snake venom metalloproteinase jararhagin. Biochem J 1996; 320:635-641.

78 Kamiguti AS, Markland FS, Zhou Q, Laing GD, Theakston RD, Zuzel M: Proteolytic cleavage of the beta 1 subunit of platelet alpha2beta 1 integrin by the metalloproteinase jararhagin compromises collagen-stimulated phosphorylation of pp72. J Biol Chem 1997;272:32599-32605.

79 Ivaska J, Kapyla J, Pentikainen O, Hoffren AM, Hermonen J, Huttunen P, Johnson MS, Heino J: A peptide inhibiting the collagen binding function of integrin alpha2I domain. J Biol Chem 1999;274:3513-3521.

80 Pentikainen O, Hoffren AM, Ivaska J, Kapyla J, Nyronen T, Heino J, Johnson MS: 'RKKH' peptides from the snake venom metalloproteinase of Bothrops jararaca bind near the metal ion-dependent adhesion site of the human integrin alpha(2)I-domain. J Biol Chem 1999; 274:31493-31505.
81 Shin Y, Morita T: Rhodocytin, a functional novel platelet agonist belonging to the heterodimeric C-type lectin family, induces platelet aggregation independently of glycoprotein Ib. Biochem Biophys Res Commun 1998;245:741-745.

82 Inoue K, Ozaki Y, Satoh K, Wu Y, Yatomi Y, Shin Y, Morita T: Signal transduction pathways mediated by glycoprotein la/lla in human platelets: Comparison with those of glycoprotein VI. Biochem Biophys Res Commun 1999;256:114-120.

83 Suzuki-Inoue K, Ozaki Y, Kainoh M, Shin Y, Wu Y, Yatomi Y, Ohmori T, Tanaka T, Satoh K, Morita $\mathrm{T}$ : Rhodocytin induces platelet aggregation by interacting with glycoprotein Ia/IIa (GPIa/IIa, integrin alpha 2beta 1). J Biol Chem 2001;276: 1643-1652.

84 Rucavado A, Soto M, Kamiguti AS, Theakston RD, Fox JW, Escalante T, Gutierrez JM: Characterization of aspercetin, a platelet aggregating component from the venom of the snake Bothrops asper which induces thrombocytopenia and potentiates metalloproteinase-induced hemorrhage. Thromb Haemost 2001;85: 710-715.

85 Hamako J, Matsui T, Suzuki M, Ito M, Makita K, Fujimura Y, Ozeki Y, Titani K: Purification and characterization of bitiscetin, a novel von Willebrand factor modulator protein from Bitis arietans snake venom. Biochem Biophys Res Commun 1996;226:273-279.

86 Chen YL, Tsai IH: Functional and sequence characterization of agkicetin, a new glycoprotein Ib antagonist isolated from Agkistrodon acutus venom. offf $2 \mathrm{p} 4$. Biochem Biophys Res Commun 1995;210:472-477.

87 Peng M, Lu W, Kirby EP: Alboaggregin-B: A new platelet agonist that binds to platelet membrane glycoprotein Ib. Biochemistry 1991;30: 11529-11536.

88 Usami Y, Suzuki M, Yoshida E, Sakurai Y, Hirano K, Kawasaki T, Fujimura Y, Titani K: Primary structure of alboaggregin-B purified from the venom of Trimeresurus albolabris. Biochem Biophys Res Commun 1996;219:727-733. 
89 Chang MC, Lin HK, Peng HC, Huang TF: Antithrombotic effect of crotalin, a platelet membrane glycoprotein $\mathrm{Ib}$ antagonist from venom of Crotalus atrox. Blood 1998;91: 1582-1589.

90 Sakurai Y, Fujimura Y, Kokubo T, Imamura K, Kawasaki T, Handa M, Suzuki M, Matsui T, Titani K, Yoshioka A: The cDNA cloning and molecular characterization of a snake venom platelet glycoprotein Ib-binding protein, mamushigin, from Agkistrodon halys blomhoffii venom. Thromb Haemost 1998;79: 1199-1207.
91 Taniuchi Y, Kawasaki T, Fujimura Y, Suzuki M, Titani K, Sakai Y, Kaku S, Hisamichi N, Satoh N, Takenaka T, et al: Flavocetin-A and -B two high molecular mass glycoprotein $\mathrm{Ib}$ binding proteins with high affinity purified from Trimeresurus flavoviridis venom, inhibit platelet aggregation at high shear stress. Biochim Biophys Acta 1995;1244:331338.

92 Fujimura Y, Ikeda Y, Miura S, Yoshida E, Shima H, Nishida S, Suzuki M, Titani K, Taniuchi Y, Kawasaki $\mathrm{T}$ : Isolation and characterization of jararaca GPIb-BP, a snake venom antagonist specific to platelet glycoprotein Ib. Thromb Haemost 1995; 74:743-750.
93 Kawasaki T, Taniuchi Y, Hisamichi N, Fujimura Y, Suzuki M, Titani K, Sakai Y, Kaku S, Satoh N, Takenaka $\mathrm{T}$, et al: Tokaracetin, a new platelet antagonist that binds to platelet glycoprotein Ib and inhibits von Willebrand factor-dependent shear-induced platelet aggregation. Biochem J 1995;308:947-953.

94 Hamako J, Matsui T, Nishida S, Nomura S, Fujimura Y, Ito M, Ozeki Y, Titani K: Purification and characterisation of kaouthiagin, a von Willebrand factor-binding and cleaving metalloproteinase from Naja kaouthia cobra venom. Thromb Haemost 1998;80:499_ 505. 